\title{
Photodynamic antibacterial action of guanidine and biguanidine derivatives of chlorin e6
}

Maria Sokolova ${ }^{1}$, Anastasia Ignatova ${ }^{2}$, Petr Ostroverkhov ${ }^{1}$, Andrey Mironov ${ }^{1}$, Mikhail Grin ${ }^{1}$ and Alexey Feofanov $^{3}$

${ }^{1}$ Institute of Fine Chemical Technology, Moscow Technological University (MIREA), Moscow, Russia, Moscow, Russia, ${ }^{2}$ Shemyakin-Ovchinnikov Institute of Bioorganic Chemistry, Russian Academy of Sciences, Moscow, Russia, Moscow, Russia, ${ }^{3}$ Biological Faculty, Lomonosov Moscow State University, Moscow, Russia, Moscow, Russia

Bacterial infections are a serious threat to health and one of the main causes of death in patients with surgical wounds or traumatic injuries. This problem is compounded by the development of bacterial resistance to antibiotics [1] and formation of bacterial biofilms that are more resistant to treatment because of reduced metabolism of bacteria and the presence of a matrix. Photodynamic inactivation of planktonic bacteria and their biofilms is considered as a promising method of treating local bacterial infections [2, 3]. Antibacterial effect is achieved by local light irradiation of lesions in the presence light-activated molecules - photosensitizers (PSs), which generate reactive oxygen species (ROS) destroying cells.

The successful development of antimicrobial photodynamic therapy (APDT) is largely determined by PS properties that makes it important to optimize PS structure in order to increase the bactericidal activity and expand the spectrum of antimicrobial action. Here we report on photodynamic antibacterial activity of chlorin e6 derivatives containing guanidine (compound 1) and biguanidine (compound 2) groups, positive charge of which should enhance the PS interaction with bacteria. The guanidine groups were attached to the chromophore via an aminoalkylamide linker, which enhances considerably accumulation of chlorin derivatives in cancer cells [4].

Compounds 1 and 2 were found to produce photodynamic bactericidal effect in the low micromolar range of concentrations against gram-positive Staphylococcus aureus 209P bacteria. The biguanidine derivative 2 possesses almost 3 times higher activity against $S$. aureus biofilms compared to 1, while activities of 1 and 2 are the same against planktonic bacteria. At the same time compounds 1 and 2 at the concentrations of $20 \mu \mathrm{M}$ and less do not affect the viability of gram-negative Escherichia coli C600 bacteria in plaktonic form or in biofilms either in the dark or under red light irradiation. To clarify a reason of insensitivity of E.coli cells to APDT, we have studied interactions of fluorescent compounds 1 and 2 with bacteria using confocal laser scanning microscopy (CLSM). 
We found that compounds 1 and 2 bind intensively to the $S$. aureus cell membrane (Fig. 1), whereas no PS binding was observed with E.coli cells (not shown). This drastic distinction in PS binding is a main reason of different sensitivity of gram-positive and gram-negative cells to APDT. ROS are short living and damage closely situated biomolecules only. In the case of E. coli cells, concentration of 1 (2) bound to cells is too low to kill them during irradiation.

Membrane localization of 1 (2) in S. aureus cells allowed us to suppose that plasma membrane is a main target of photodynamic action of the studied PS. To verify this hypothesis, S. aureus cells were incubated with 1 (2), hydrophobic acetoxymethyl ester of BCECF and membrane impermeable propidium iodide (PI). Within cells, acetoxymethyl ester of BCECF is cleaved to highly charged fluorescent BCECF, which is not able to penetrate through plasma membrane (Fig. 2 A). Irradiation of bacteria preincubated with 2 or $1(5 \mu \mathrm{M}$ for $1 \mathrm{~h})$ resulted in the immediate BCECF leakage from cells and intracellular PI accumulation (Figure 2B, D) that evidences photoinduced disintegration of the plasma membrane.

In conclusion, compounds 1 and, especially, 2 are efficient PS for APDT of gram-positive bacteria, but their structure requires further optimization to enhance binding to and photoinduced damage of gramnegative bacteria.

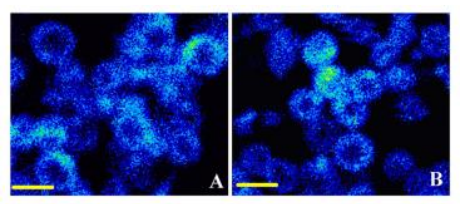

Figure 1. Confocal images showing distribution of compounds 1 (A) and 2 (B) in S. aureus cells. Cells were incubated with $1 \mu \mathrm{M}$ of compounds 1 (A) or 2 (B) for $1 \mathrm{~h}$. Fluorescence was detected in the >650 nm spectral range. The bar size is $1 \mu \mathrm{m}$.

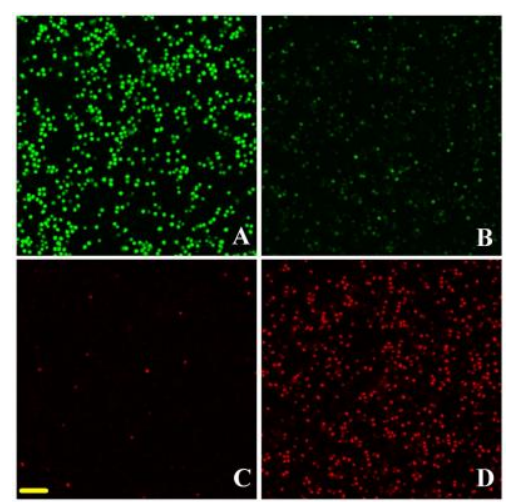

Figure 2. Bactericidal effect of 2 is related to permeabilization of the bacterial membrane. Confocal images of S.aureus bacterial biofilms stained by BCECF and PI are shown: (A, C) intact cells; (B, D) cells pre-incubated with $5 \mu \mathrm{M}$ compound 2 and irradiated. (A, C) BCECF fluorescence recorded in the 500$550 \mathrm{~nm}$ spectral range. (C, D) PI fluorescence recorded in the 650-700 nm range. The bar size is $6 \mu \mathrm{m}$. 
Images $\mathrm{A}$ and $\mathrm{B}, \mathrm{C}$ and $\mathrm{D}$ were recorded at the same parameters and can be directly compared by the signal intensity. Leakage of BCECF from cells accompanied by PI accumulation in cells indicates membrane permeabilization. BCECF - 2',7'-Bis-(2-carboxyethyl)-5-(6)- carboxyfluorescein. PI propidium iodide.

References

[1] Antibiotic Resistance. Available online: https://www.who.int/news-room/fact-sheets/detail/antibioticresistance

[2] A. Azaripour et al. Lasers Med Sci. 33(2) (2018) P. 407-423.

[3] G.B. Kharkwal et al. Lasers Surg Med. 43(7) (2011) P.755-767.

[4] A.V. Efremenko et al. Photochem. Photobiol. Sci. 13 (2014) P. 92-102.

[5] The financial support of RFBR grant 19-04-00854 is acknowledged. 